\title{
Surgical Menopause
}

National Cancer Institute

\section{Source}

National Cancer Institute. Surgical Menopause. NCI Thesaurus. Code C94256.

Menopause that is induced by the surgical removal of the ovaries. 